\title{
OPTIMIZING NETWORK TRAFFIC AND DATA SEARCH EFFICIENCY AS MOBILE CLOUD SERVICE
}

\author{
Ahmed Hussain ${ }^{1}$, Shimi Jeyaseelan ${ }^{2}$ \\ ${ }^{I}$ M.tech (Student), T.John Institute of Technology \\ ahmedpride123@gmail.com \\ ${ }^{2}$ Assistant Professor, T.John Institute of Technology \\ shimi.james@gmail.com
}

\begin{abstract}
Report stockpiling in the cloud association is quickly snap power all through the world. In any case, it position threat to clients unless the data is encoded for security division. Blended data ought to be viably searchable and retrievable with no certification spills, especially for the advantageous customer. the settled different security the issues, the blueprint can't be related on cell telephones unmistakably under the adaptable cloud environment. This is an immediate determination of the difficulties obliged by remote fabrics, for case, lethargy affectability, poor framework,. This prompts a long demand fourth measurement and lance bearer structure development costs while utilizing standard pursue orchestrates. This work reference these issues by proposing a profitable Encrypted Data Search (En-das) plan as an advantageous cloud association. This innovative course of action uses a lightweight trapdoor (encoded catchphrase) weight technique, which pushes the information correspondence process by decreasing the trapdoor's size for ontogeny capacity. In this study, we additionally propose two streamlining approaches for record search for, Trapdoor Mapping Table (TMT) Serial Binary Search (RSBS) calculation, to stop number the solicitation time. Results demonstrate En-das reduces search for time by and what's more structure improvement.
\end{abstract}

Keywords: En-das, Look Up, Trap Door

\section{INTRODUCTION}

Since dispersed figuring can bolster adaptable associations and give a sparing utilization of point of confinement and number assets, it is quickly getting notoriety. With outstanding cloud associations, different information suppliers can populate their information in fogs rather than especially serving clients. The cloud likewise permits suppliers to name key errands, for occasion, report searches for. To ensure information, the archives and records ordinarily blended before outsourcing to the cloud for hobbies. Right when clients need to investigate certain documents, they watch words to essential information supplier. The supplier makes blended watchwords and gives back the trap-door to the client. The client then sends these to cloud. In the wake of enduring the trap-door, the Cloud utilizes a remarkable solicitation figuring to pick a strategy of required reports (encoded) considering the blended records and given trapdoor. At last, the client gets these blended inquiry things and utilizations the private key from the supplier to unwind archives, ensures information security while qualifying the suppliers for use both the tally and farthest point force of the Cloud for record looks. Because of these great circumstances, this planning has beginning now been all around got in security guaranteeing pursue structures.
Telephones pushed cell phones were reviewed to surpass. These days, clients vivaciously use telephones to demand report search for associations. As a rule, telephones accomplice with the Internet by and large by strategy for remote systems (Wi-Fi/3G/4G/LTE), which secures a few inconveniences when showed up distinctively in connection to routine wired structures. These inconveniences include:

Latency affectability: these remote structures accomplish longer system idleness, which can back off a solitary hobby if the solicitation requires different structure round excursions. For instance, in the standard structure appeared in solitary hobby requires 3 round treks results in acclaimed stillness remote correspondence.

Poor framework: gadgets are reliably unequipped for keeping up a long running association with the Cloud, by and large for vitality sparing purposes. Distinctive pursue deals could realize diverse reassociation operations and additional acknowledge-

ment expenses.

Low system transmission rate: Mobile contraptions are ordinarily outfitted with control transmission segments, rates of slower sending. 


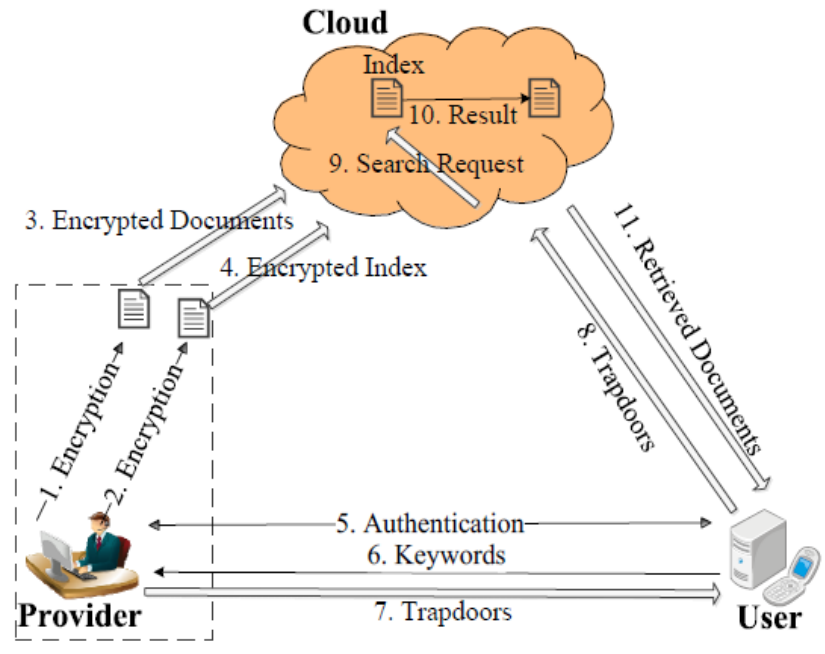

Fig 1.1 Usual cloud search using encryption

For instance, the standard structure appeared requires 2 system circle treks between the client and the supplier and one between the client and the cloud. Three round just compel conspicuous solicitation delay and over the top system improvement, which could be excessive for a cellular telephone

\section{EXISTING SYSTEMS}

Precisely when clients need to examine certain archives, they first send watchwords to the primary information supplier. The supplier then conveys blended catchphrases and gives back the trap-door to the client. The client then sends these trap-doors to the cloud. Ensuing to getting the trap-door, the Cloud utilizes a momentous pursue number to pick a strategy of fancied docs(blended) in light of the encoded records and given trap-door. At long last, the client gets these blended once-over things and utilizations the private key from the supplier to translate reports. This layout, as portrayed in Fig 1.1, ensures information security while qualifying the suppliers for use both the tally and breaking point force of the Cloud for document looks. In perspective of these positive circumstances, this planning has beginning now been all around got a handle on in certification protecting search for frameworks.

\section{Disadvantages}

- Look for postponement.

- Over the top structure advancement.

- Costlier for cellular telephone.

- More transmission point of confinement use. the trapdoor should be transmitted 2 Time per demand

\section{PROPOSED SYSTEM}

In this I concentrate on advancement \& time of interest wastefulness issues regarding minimized cloud. We demonstrate a skilled Scrambled Information Look (En-das) plan as a helpful cloud association to handle these issues. Our structure underpins multi-watchword security protecting look and hugely decreases system development and pursue delays. For system development, En-das pre-figures trapdoor for general interest catchphrases and along these lines stays away from one structure round trip for re-handling trapdoor per demand. We put forth a few sections to pack trap-door and show that our pre-enlisted trap-door and could attainably secured in telephone. As to time, En-das retrofits the interest calculation in the cloud. In light of the coordinated tree standard, we show Positioned Serial Parallel Inquiry (RSBS) estimation, which could decrease question time in the cloud.

\section{Advantages}

The benefits of the proposed structure are:-

- En-das enhances structure activity and pursue time benefit separated and the standard framework.

- Minimal using of cloud is done in En-das

- Network development is diminished by a solitary round trip data trade and the trap-door weight strategy thinking.

- The search for value is lessened by the figuring the TMT.

\section{SYSTEM DESIGN}

\section{- Experiment environmental.}

Experimental occurrence should be as we think son that procedure can be done as we want. Trap-door, TMT and RSBS is used to go through our things. Systems with nice configuration are used with cloud and other are system disks and c p u. trap - door is processed first on system \& then shifted to phone.

\section{- Search Time Evolution.}

Decreasing the inquiry time to enhance the figuring productivity, we used the TMT \& RSBS working on En-das square work. The 1 st part, we assess to the general inquiry time to its divisions. At that point we introduce the execution or the RSBS calculation as far as the pursuit time

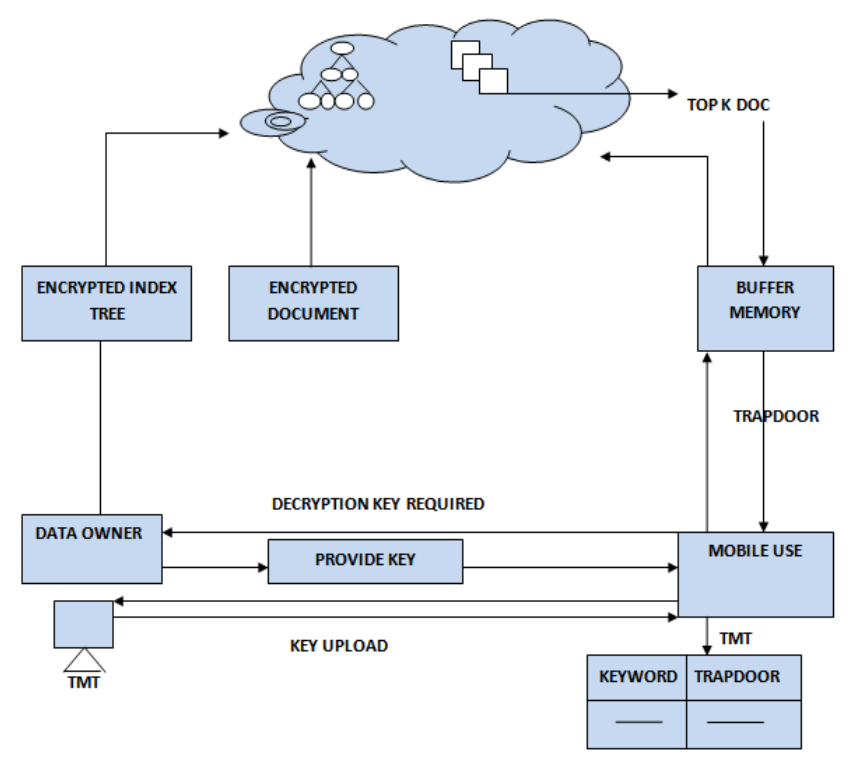

Fig 1.2 System Architecture 


\section{- Network Traffic Evolution.}

En-das framework, which profits by the trap-door pressure technique \& to the $\mathrm{T} \mathrm{M} \mathrm{T} \mathrm{things,} \mathrm{we} \mathrm{decreased} \mathrm{system}$ move12ment fundamentally. Next we assess and break down the general framework system activity diminishment and the execution of the trapdoor pressure strategy.

\section{Retrofitted Trap-door Generation Process}

This gives the detailed procedure of the trap-door its working and all the minute detailing is told here and elaborated properly

Below algorithm gives all calculation and other things done in this procedure

Trap door generation

Step 1: input keyword $\mathrm{k}$

Step 2: Hash function of FAH algorithm

Step 3: Mapping of FAH algorithm

Step 4: Index compressed trap door

Step 5: Extract term t from K

Step 6: pure trap door without noise is obtained

Step 7: Location is calculated and recorded the accumulated value.

It is attractive to store information on information stockpiling servers, for example, mail servers and record servers in scrambled structure to decrease security and protection dangers. In any case, this as a rule suggests that one needs to give up usefulness for security. For instance, if a customer wishes to recover just reports containing certain words, it was not already known how to give the information stockpiling server a chance to perform the hunt and answer the inquiry, without loss of information secrecy. We portray our cryptographic arrangements for the issue of looking for on mixed data and give proofs of security to the resulting crypto structures. Our systems have different basic central focuses. They are provably secure: they give provable secret to encryption, as in the untrusted server can't learn anything about the plaintext when simply given the cipher text; they give request isolation to request, inferring that the untrusted server can't learn much else about the plaintext than the yield; they give controlled looking, so that the untrusted server can't chase below an optional word without the customer's authorization; they additionally bolster concealed questions, so that the client may approach the untrusted server to hunt down mystery word without uncovering the word to the server. The calculations exhibited are straightforward, quick (for a report of length n, the encryption and inquiry calculations just need $\mathrm{O}(\mathrm{n})$ stream figure and piece figure operations), and present no space and correspondence overhead, and henceforth are commonsense to utilize today.

\section{RESULTS}

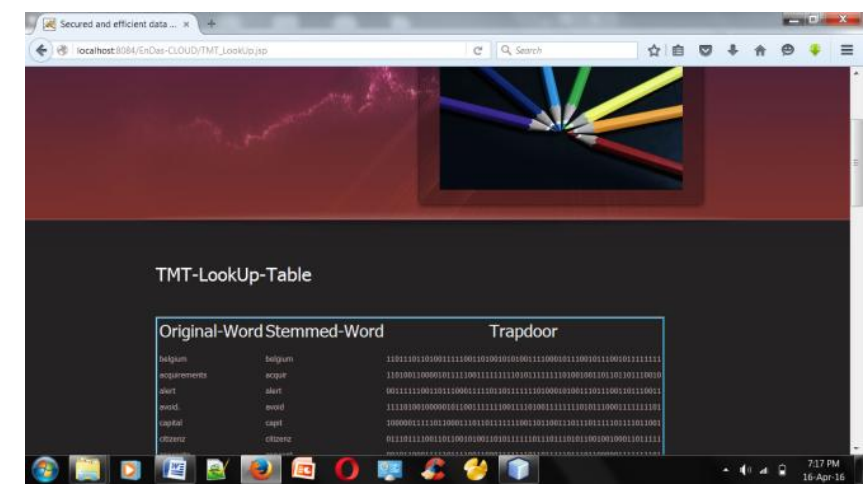

Fig 1.3 Lookup table

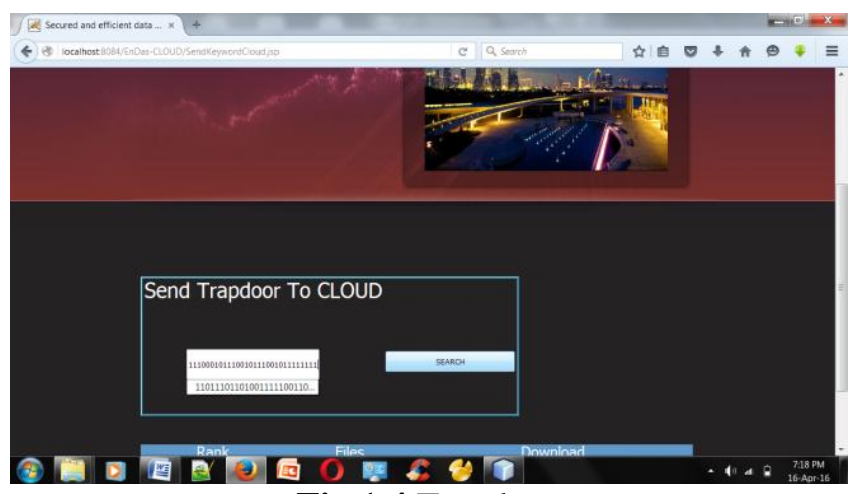

Fig 1.4 Trapdoor

\section{CONCLUSIONS}

Proposing a novel look forward framework En-das over the versatile cloud, this enhances system development and chase time capability contrasted and the standard structure. We started with an intensive investigation of the standard encoded look for system and dissected its bottlenecks in the versatile cloud: system activity and look time inefficiency. By then we built up a capable configuration of En-das which is suitable for the versatile cloud to address these issues, where we used the TMT and the RSBS figuring to adapt to the inefficient chase time issue, while a trapdoor pressure system was used to reduce system movement costs. Finally our evaluation concentrate tentatively shows the execution central purposes of EnDAS Client information would be stored utilizing support memory. At the point when the client sign in and hunt down the information taking into account watchwords, the information in the cloud which would be stored. At the point when some other client will be seeking with the same watchwords can be served from the reserved which makes the looking system proficient.

\section{REFERENCES}

[1]. Towards Statistical Queries over Distributed Private User Data Ruichuan Chen† Alexey Reznichenko† Paul Francis $\dagger$ Johannes Gehrke $§$ †Max Planck Institute for Software Systems (MPI-SWS), Germany §Cornell University, Ithaca, NY 14853, USA

[2]. Efficient and Secure Ranked Multi-Keyword Search on Encrypted Cloud Data 
Cengiz Örencik Faculty of Engineering \& Natural Sciences

Sabanci University, Istanbul, 34956, Turkey cengizo@sabanciuniv.edu Erkay Sava s Faculty of Engineering \& Natural Sciences Sabanci University, Istanbul, 34956, Turkey erkays@ @abanciuniv.edu

[3]. Towards Statistical Queries over Distributed Private User Data -Ruichuan Chen, Alexey Reznichenko, Paul Francis, Johannes Gehrke.

[4]. Fast accumulated hashing Kaisa Nyberg.

[5]. On the Design and Security of Block Ciphers A dissertation submitted to the SWISS FEDERAL INSTITUTE OF TECHNOLOGY ZURICH

[6]. Privacy-Preserving Multi-keyword Ranked Search over Encrypted Cloud Data Author: -Ning Cao, Cong Wang, Ming Li, Kui Ren, and Wenjing Lou

[7]. Towards Statistical Queries over Distributed Private User Data Author: -Ruichuan Chen, Alexey Reznichenko, Paul Francis, JohannesGehrke.

[8]. Secure Indexes Author: -Eu-Jin Goh

[9]. Privacy-Preserving Multi-Keyword Fuzzy Search over Encrypted Data in the Cloud Author: -Bing Wang, Shucheng YuyWenjing Lou, Y. Thomas Hou.

[10]. Privacy Preserving Keyword Searches on Remote Encrypted Data Author: -Yan-Cheng Chang and Michael Mitzenmacher.

[11]. Zerber: r-Confidential Indexing for Distributed Documents Author: -SergejZerr, Elena Demidova, Daniel Olmedilla, Wolfgang Nejd. 